\title{
Resolving semantic and proactive interference in memory over the short-term
}

\author{
Alexandra S. Atkins • Marc G. Berman • \\ Patricia A. Reuter-Lorenz • Richard L. Lewis • \\ John Jonides
}

Published online: 15 February 2011

(C) Psychonomic Society, Inc. 2011

\begin{abstract}
Interference is a major source of short-term errors of memory. The present investigation explores the relationship between two important forms of interference: proactive interference (PI), induced by the need to reject recently studied items no longer relevant to task performance, and semantic interference (SI), induced by the need to reject lures sharing a meaningful relationship with current memoranda. We explore the possibility that shared cognitive control processes are recruited to resolve both forms of interference. In Experiment 1 , we find that the requirement to engage in articulatory suppression during the retention interval of tasks that induce either PI or SI increases both forms of interference similarly and selectively. In Experiment 2, we develop a task to examine PI and SI within the same experimental context. The results show interactive effects between factors that lead to the two forms of interference. Taken together, these findings support contextual-cuing models of short-term remembering (Nairne, Annual Review of Psychology, 53, 53-81 2002), where the context in which retrieval occurs can influence susceptibility to interference. Lastly, we discuss several theoretical hypotheses concerning the cognitive control processes that are recruited to resolve SI and PI in shortterm remembering.
\end{abstract}

\footnotetext{
A. S. Atkins $(\bowtie)$

Duke University,

LSRC Building, B203, Box 90999, Durham, NC 27708-0999, USA

e-mail: alex.atkins@duke.edu
}

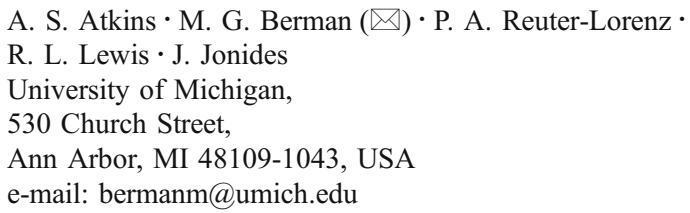

Keywords Interference $\cdot$ Short-term memory $\cdot$ Working memory $\cdot$ False memory $\cdot$ Cognitive control

Interference is one of the greatest burdens on the cognitive system. It is the reason we put the cereal box in the refrigerator when we have an idle thought, but it is also related to more serious memory failures, which can lead to significant accidents. Interference has profound adverse affects on short-term remembering, and it is one of the major sources of forgetting over brief retention intervals (Berman, Jonides, \& Lewis, 2009; Lewandowsky, Geiger, \& Oberauer, 2008; Nairne, 2002; Wixted, 2005).

Memory is susceptible to multiple forms of interference. Classic investigations of episodic learning have demonstrated both proactive interference (PI) effects, wherein memory for previously learned information interfered with new learning, and retroactive interference effects, wherein new learning interfered with memory for previously learned associations (see Keppel, 1968; Postman \& Underwood, 1973). Later research on false memory demonstrated that episodic memory is vulnerable to remarkable semantic distortion, such that participants will falsely recall and recognize items related in meaning to previously studied memoranda (see, e.g., Roediger \& McDermott, 1995).

Regarding remembering over short intervals, limited to several seconds, early work demonstrated that items that are acoustically and visually similar to memoranda are often substituted for studied items at retrieval (Conrad, 1964; Laughery \& Harris, 1970; Logie, Della Sala, Wynn, \& Baddeley, 2000). Early work also established that similar semantic contexts lead to a buildup of interference. In classic release-from-PI paradigms (cf. Wickens, 1970), episodic recall and recognition of items in a current memory set declines as a function of the number of 
previous lists studied from the same semantic category. When the category is changed, PI is reduced, suggesting a release from interference with a change in semantic context (Wickens, 1970; Wickens, Dalezman, \& Eggemeier, 1976).

More recent investigations have highlighted the strong influences of both episodic (temporal) familiarity and semantic context on short-term retrieval. With respect to episodic familiarity, the dominant task used to assess interference effects is the recent-probes task (Berman et al., 2009; Jonides, Smith, Marshuetz, Koeppe, \& Reuter-Lorenz, 1998; Monsell, 1978). In this task, participants study a set of items that are retained for several seconds. After the retention interval, subjects are presented with a probe to which they must make a yes/no response. Critically, "no" probes are of two kinds: recent-no and nonrecent-no. Recentno probes are items that are not present on the current trial but were present in the memory set on the trial immediately preceding the current one; nonrecent-no items are probes that did not appear on the last several trials. Investigations of the recent-probes task consistently show that participants are markedly slower and more error-prone when rejecting recent-no probes relative to nonrecent-no probes. These findings have been interpreted as evidence for the need to engage in interference resolution, a controlled cognitive process by which the episodic familiarity of recent-no probes is adjudicated in the service of accurate task performance (Jonides \& Nee, 2006).

Regarding semantic context, recent work has demonstrated semantic interference (SI) effects in short-term remembering by showing that rejection of unstudied items related in meaning to those in memory is both slower and more error-prone than rejection of semantically unrelated items (Atkins \& Reuter-Lorenz, 2008). For example, Atkins and Reuter-Lorenz (2008) investigated false recognition of semantic lures using a short-term variant of the Deese/ Roediger-McDermott (DRM) paradigm (Roediger \& McDermott, 1995). In this task, participants studied sets of four words, which were all associated with a common, unstudied theme word. Following a 3- to 4-s retention interval, which was either unfilled or filled with a distracting math verification task, participants saw a single probe word to which they made a yes/no response to indicate whether the probe was a member of the current memory set. "No" probes were of two kinds: unrelated-no probes, which were not members of the current set and were unrelated in meaning to the memorized items, and related-no (lure) probes, which consisted of the unstudied theme word associated with the items in the current set. Findings showed reliable false recognition of lure probes and high levels of interference associated with correct rejection of these items relative to unrelated "no" probes. This suggests that semantic context, like episodic recency, can induce interference in short-term remembering.
Taken together, the findings regarding interference effects and semantic distortion in episodic remembering, coupled with demonstrations of PI and SI in canonical short-term memory tasks (such as the recent-probes and short-term DRM paradigms), suggest that similar forms of interference may influence memory over short- and longterm intervals. Although they are inconsistent with strict multi- and dual-store models of memory (e.g., Atkinson \& Shiffrin, 1968; Baddeley \& Hitch, 1974), parallel interference effects across short-term and episodic remembering are fully consistent with interference-based theories of memory, such as those of Postman (1961) and Underwood and Schultz (1960), and with modern activation-based accounts that conceptualize short-term memory representations as temporarily active long-term memory representations (Anderson et al., 2004; Cowan, 1988, 1995, 2000; McElree, 2001; Nichols, Kao, Verfaellie, \& Gabrieli, 2006; Oberauer, 2002; Ranganath \& Blumenfeld, 2005; Ranganath \& D'Esposito, 2005; Verhaeghen, Cerella, \& Basak, 2004; for a review, see Jonides et al., 2008). Although such models can accommodate the findings of both PI and SI in short-term remembering, they remain agnostic regarding the role of cognitive control in interference resolution. Furthermore, there is an open question regarding the extent to which these two forms of interference may interact to influence memory over the short term.

The present work addressed these issues by directly examining the relationship between PI and SI in short-term remembering and examining the extent to which both forms of interference are mediated by shared cognitive control processes. In a related experiment, Oztekin, Curtis, and McElree (2008) looked at the relationship between resolving PI and SI both neurally and behaviorally, by examining the buildup of PI. ${ }^{1}$ Our work builds on their findings, by independently manipulating PI and SI. In doing so, we were able to assess both the independent effects of and the interactions between PI and SI.

Experiment 1 examined the effects of articulatory suppression during the retention intervals of both the recent-probes task and the short-term DRM task. We hypothesized that articulatory suppression would interfere with verbatim rehearsal processes (Baddeley, Thomson, \& Buchanan, 1975; Levy, 1971), thus reducing the distinctiveness of current memoranda by decreasing the signal-tonoise ratio of these items relative to recent-no or lure probes in both tasks. If so, articulatory suppression should selectively increase both PI and SI, increasing false alarms to and slowing correct rejections of recent-no items in the recent-probes task, as well as increasing false alarms to and

\footnotetext{
${ }^{1}$ In Oztekin et al. (2008) experiment, there was semantic interference on every trial, since every probe was semantically related to the memory set.
} 
slowing correct rejections of related-no (lure) items in the short-term DRM task. Results from Experiment 1 supported these predictions and are consistent with the hypothesis that PI and SI tasks utilize shared resources.

Experiment 2 tested the relationship between PI and SI more directly by using a novel variant of the recent-probes task that incorporates semantically categorized memoranda. This procedure permitted the concurrent assessment of PI and SI within subjects. The results demonstrate an interaction between PI and SI and suggest that the resolution of both forms of interference may recruit shared cognitive control processes. The type and nature of these shared processes are discussed in the General Discussion. In addition, the relationship between these findings and contextual-cuing-based models of short-term memory (e.g., Nairne, 2002) is considered.

\section{Experiment 1}

The purpose of Experiment 1 was to test whether engagement in articulatory suppression during the retention intervals of the recent-probes and short-term DRM tasks would influence the magnitude of proactive and semantic interference effects. We hypothesized that this manipulation would increase both PI and SI by reducing the distinctiveness of memoranda and recent-no items, which are episodically familiar, and related-no items, which share a semantic context with the memorized items. Support for this hypothesis would provide preliminary evidence that similar processes may be required to overcome both PI and SI, and that the efficacy of these processes is reduced by articulatory suppression. Critically, we hypothesized that articulatory suppression would disproportionately affect the interference trials in both tasks rather than simply worsening overall performance.

Method

\section{Participants}

Thirty-two participants were recruited from the University of Michigan. All participants gave informed consent, as reviewed by the university's Institutional Review Board. Participants were paid $\$ 15$ per hour for their participation, plus bonuses for fast and accurate responding throughout the experiment. Bonus scores were calculated on a trial-bytrial basis with the following equation:

Trial Score $=$ Probe $\_$ACC $\times\left(700 \mathrm{~ms}-\right.$ Probe $\_$RT $)$,

where probe accuracy (ACC) is a binary variable, 1 if correct and 0 if incorrect, and $\mathrm{RT}$ is response time.
Individual trial scores were summed together to yield a total score. The participants were paid a penny for each point of their total score.

\section{Design/procedure}

To examine the effects of articulatory suppression on PI and SI, we utilized a 2 × 2 mixed design, with interference type (PI vs. SI) as a between-subjects variable and articulatory suppression as a within-subjects variable. As such, half of all participants $(n=16)$ completed the recent-probes task (to assess PI), and half ( $n=16)$ completed the short-term DRM task (to assess SI). Participants in each group completed two task blocks, one with articulatory suppression and one without. The order of articulatory-suppression and no-articulatory-suppression blocks was counterbalanced across participants.

Recent-probes task The recent-probes task is presented in Fig. 1a. On each trial, a set of four words was displayed for 2,000 ms. Following a variable length 3,000- to 4,000-ms retention interval, a single probe word was displayed. Participants needed to respond affirmatively if the probe was one of the words in the set, and negatively if not. The "no" trials were of interest and came in two forms. The first type was a recent-no trial, in which the probe was not a member of the current set, but was a member of the previous set. The second type was a nonrecent-no trial, in which the probe was neither a member of the current set nor a member of the two previous sets. Previous work using this task has shown that participants are both delayed and less accurate when responding to recent-no probes as compared to nonrecent-no probes, and this difference in performance is a measure of PI. We also utilized two forms of "yes" probes: recent-yes, which were present on both the current and the immediately preceding memory set, and nonrecent-yes, which were present in the current set but not in the two preceding trials. Participants completed a total of 192 recent-probes trials, including 48 instances of each of the four probe types.

Short-term DRM task The short-term DRM paradigm (Atkins \& Reuter-Lorenz, 2008) is presented in Fig. 1b. The progression of each trial was similar to that of the recent-probes task, except that memory sets consisted of four semantically related items, all associated with a common theme word. As in the recent-probes task, participants made a yes/no judgment in response to a memory probe presented following the retention interval. During this task, theme words served as the probes on all of the trials. As in the recent-probes task, there were two variations of "no" trials. The first were unrelated-no trials, in which the probe consisted of a nonpresented theme word 


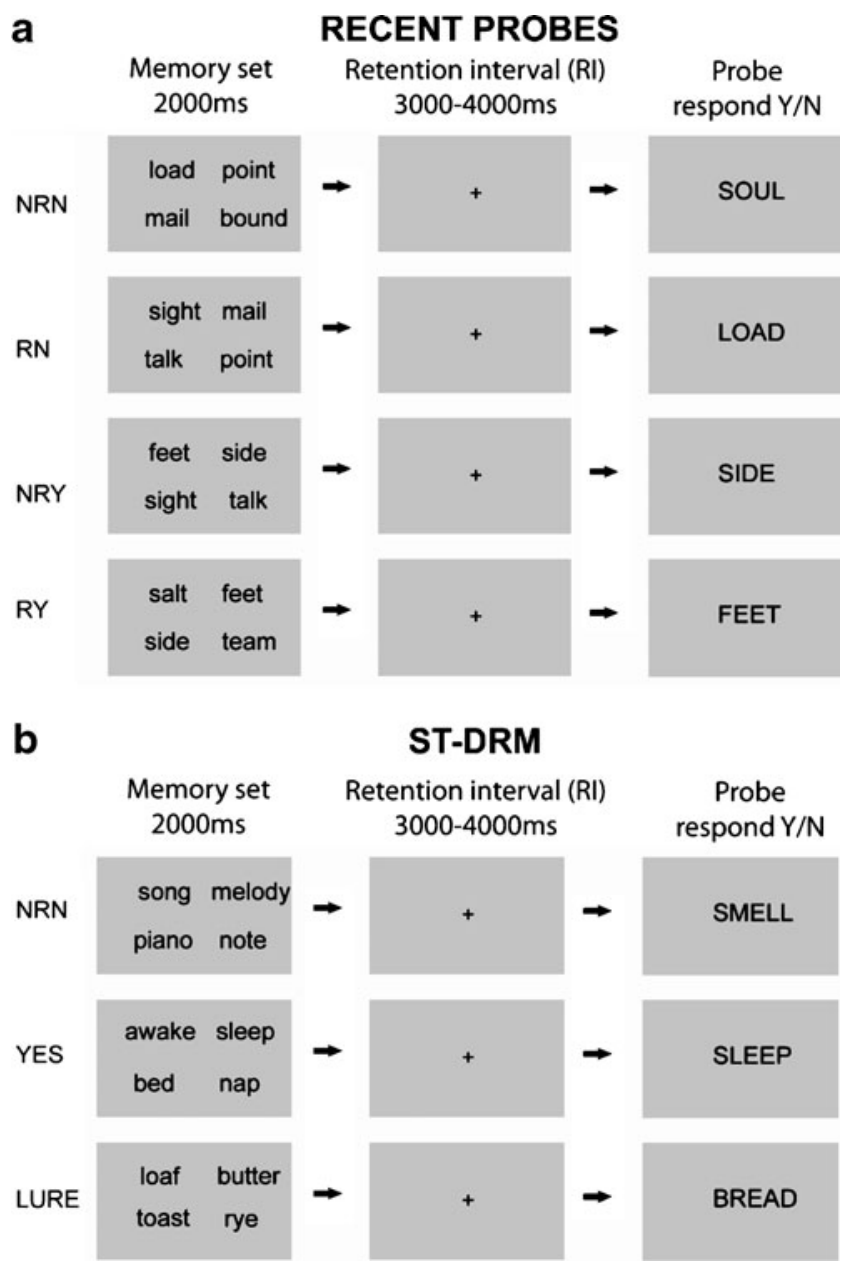

Fig. 1 The recent-probes and short-term DRM (ST-DRM) tasks used in Experiment 1. (a) In the recent-probes task, four types of recognition probes were used: nonrecent-no $(\mathrm{NRN})$, recent-no $(\mathrm{RN})$, nonrecent-yes (NRY), and recent-yes (RY) probes. (b) In the ST-DRM task, three types of probes were used: nonrelated-no (NRN), yes, and related-no (LURE) probes. During articulatory-suppression blocks for both tasks, the fixation point presented during the retention interval was bright blue rather than black. Participants began counting aloud from 1 to 3 repeatedly at the onset of this symbol and continued throughout the retention interval. See the text for details. The first row represents Trial 1, the second row Trial 2, and so forth

associated with an unstudied (i.e. never presented) list. The second were related-no trials (also referred to as lure trials), in which the probe consisted of the (unstudied) theme word associated with the present memory set. On "yes" trials, the associated theme word was embedded in the memory set and served as the "yes" probe. No participant was exposed to a given theme or probe more than once during the experiment. The mean backward associative strength, a normed measure of the semantic association between each memorandum and the theme word (see Hancock \& Hicks, 2002; Roediger, Watson, McDermott, \& Gallo, 2001), was equated across lists. Probe type was counterbalanced with lists across participants. This procedure ensured that participants encountered the same probes (all theme words), but in different contexts: as lures, unrelated-no probes, or "yes" probes. Each participant completed 51 randomly ordered short-term DRM trials, which included 17 instances of each of the three probe types.

Articulatory suppression On articulatory-suppression blocks, participants counted aloud from 1 to 3 repeatedly at a rate of approximately four utterances per second. Counting began at the onset of a blue fixation cross that appeared just after encoding and stayed on the screen throughout the retention interval. The pace of counting was practiced prior to completion of the articulatory-suppression trial block. During the experiment, counting was monitored to ensure compliance with task instructions. Articulatorysuppression and no-articulatory-suppression blocks were administered in a counterbalanced order across participants.

Results and discussion

The results from Experiment 1 were consistent with our predictions and indicate that interrupting rehearsal of items in the present memory set increases interference elicited by (1) recent-no probes, which were previously studied but are irrelevant to the current trial, and (2) related-no (lure) probes, which were never studied but have become active through spreading semantic activations.

Mean accuracy (displayed as hits and false alarms) and median response times (RTs) for correct responses to each probe type in each task are presented in Fig. 2. In order to minimize the influence of outliers in our RT analyses, median RTs for each probe type were computed for each participant; mean group RT measures were computed from these medians.

\section{Proactive interference}

Mean accuracy (displayed as hits and false alarms) and median RTs for the recent-probes task are presented in Figs. 2a and b, respectively. In order to examine the effects of probe recency and articulatory suppression in the recentprobes task, $2 \times 2 \times 2$ mixed effects ANOVAs were conducted separately for "yes" and "no" probes. The withinsubjects variables were probe recency (recent vs. nonrecent) and articulatory-suppression condition (articulatory suppression vs. no articulatory suppression). Task order (articulatory suppression first vs. articulatory suppression second) was included as a between-subjects variable.

With respect to "no" probes, we found main effects of probe recency on both false alarm rates, $F(1,14)=33.11, p<.001$, $\eta^{2}=.70$, and RTs for correct rejections, $F(1,14)=53.19, p<$ $.001, \eta^{2}=.79$, indicating that participants were both slower to 
Fig. 2 Mean accuracy (hits and false alarms) and median RTs (correct trials only) for the recent-probes task (panels a and b) and the ST-DRM task (panels c and d) used in Experiment 1. Articulatory supression (AS) reliably increased the accuracy and RT indices of both proactive interference and semantic interference

\section{a}
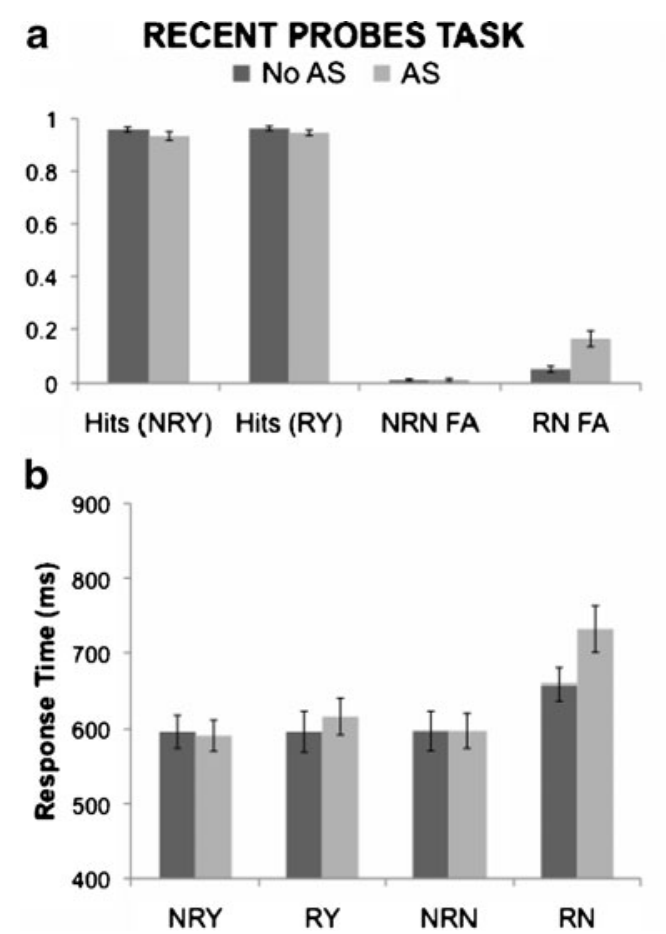
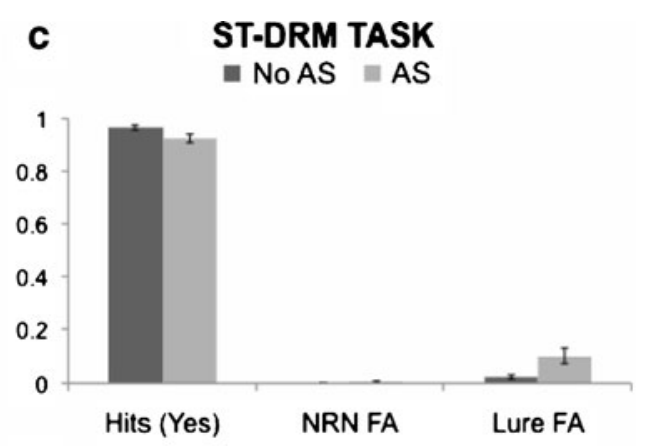

d

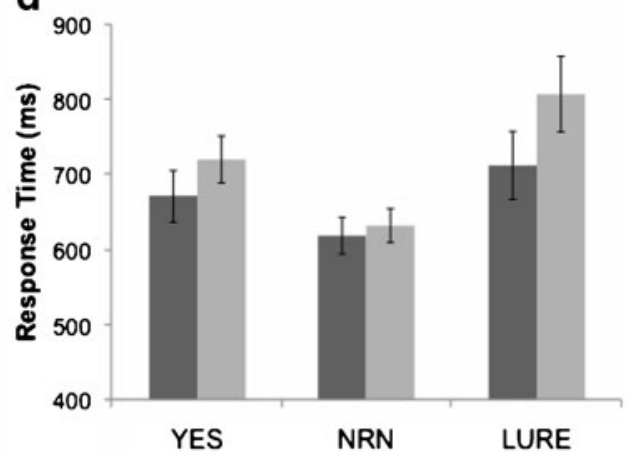

correctly reject and more likely to falsely endorse recent-no relative to nonrecent-no probes. These findings replicate others that have demonstrated PI using similar paradigms, and indicate that our task succeeded in inducing PI. Critically, we found a significant Probe Recency $x$ Articulatory Suppression Condition interaction with respect to both false alarm rates, $F(3,12)=13.86, p<.005, \eta^{2}=.50$, and RTs for correct rejections, $F(3,12)=42.48, p<.001, \eta^{2}=.75$, indicating that PI effects on both dependent measures increased with the requirement to engage in articulatory suppression. Our RT index of PI (recent-no - nonrecent-no) increased from a mean of $61 \mathrm{~ms}$ in the no-articulatorysuppression condition to a mean of $134 \mathrm{~ms}$ in the articulatorysuppression condition. Our error measure of PI, calculated as the difference in false alarm rates for recent-no and nonrecent-no probes, similarly increased from .04 in the noarticulatory-suppression condition to .15 in the articulatorysuppression condition.

Collapsing across recent-no and nonrecent-no probes, articulatory suppression was associated with increased false alarm rates, $F(1,14)=13.27, p<.005, \eta^{2}=.49$, but not with reliable differences in RTs for correct rejections $(p>.1)$. Similarly, with respect to "yes" probes, articulatory suppression was associated with reduced hit rates, $F(1,14)=$ $8.65, p<.05, \eta^{2}=.38$, but not with increases in RTs $(p>.1)$.

An examination of RTs (Fig. 2b) shows that articulatory suppression selectively increased the time required to correctly reject recent-no probes, since RTs increased only for this trial type. Post-hoc paired $t$ tests showed no reliable increase in RTs for any other probe type. In addition, articulatory suppression disproportionally affected the false alarm rate for recent-no trials as compared to the other probe types. Although hit rates for nonrecent-yes and recent-yes trials were reduced by articulatory suppression, the effect was reliably stronger for the false alarm rate for recent-no trials (there was no change in false alarms to nonrecent-no probes). In sum, these results indicate that articulatory suppression disproportionally affected recent-no probes (the trials that had PI) and was not associated with a general slowing of responses or with worse overall accuracy.

\section{Semantic interference}

Mean accuracy (displayed as hits and false alarms) and median RTs for the short-term DRM task are presented in Figs. 2c and d, respectively. Repeated measures ANOVAs were conducted to assess the effects of "no" probe type (unrelated-no vs. lure), articulatory suppression condition, and task order on both dependent measures. Participants showed increased false alarms to related-no, relative to unrelated-no, probes, $F(1,14)=25.20, p<.001, \eta^{2}=.64$. Furthermore, correct rejections of related-no probes were slowed relative to unrelated-no probes, $F(1,14)=29.01$, $p<.001, \eta^{2}=.67$. These main effects replicate our previous work (Atkins \& Reuter-Lorenz, 2008) and demonstrate the effectiveness of the short-term DRM task in producing reliable measures of SI and false recognition.

Main effects of articulatory suppression were found with respect to both false alarm rates, $F(1,14)=6.26, \mathrm{P}<.05, \eta^{2}=$ .31 , and RTs for correct rejections, $F(1,14)=10.70, p<.05$, $\eta^{2}=.31$. Thus, collapsing across related-no and unrelated-no 
probes, participants were both more error prone and slower to correctly respond in the articulatory-suppression block. Critically, however, we found reliable "No" Probe Type (unrelated-no vs. related-no) x Articulatory-Suppression Condition interactions on both false alarm rates, $F(3,12)=$ 5.81, $p<.05, \eta^{2}=.29$, and RTs for correct rejections, $F(3$, 12) $=8.42, p<.05, \eta^{2}=.34$, indicating that both accuracy and RT measures of SI disproportionally increased with articulatory suppression. Our RT index of SI (related-no unrelated-no) increased from a mean of $92 \mathrm{~ms}$ in the noarticulatory-suppression condition to a mean of $173 \mathrm{~ms}$ in the articulatory-suppression condition. Our error measure of SI, calculated as the difference in false alarms to unrelatedno versus related-no probes, also increased, from .02 in the no-articulatory-suppression condition to .10 in the articulatory-suppression condition.

These results indicate significant effects of articulatory suppression on responses to related-no items. With respect to RT, engaging in articulatory suppression reliably increased RTs to correctly rejected related-no probes (lures) only. There was no effect of articulatory suppression on RTs for either correct rejections of unrelated-no probes (NRN) or correct recognition of "yes" probes (hits). Articulatory suppression also increased the false alarm rate for related-no probes and reduced the hit rate, but did not affect the false alarm rate to unrelated-no probes. Although articulatory suppression was associated with a reduction in hits, this effect was marginally smaller than the increase in false alarms to related lures. Therefore, as was true in the recent-probes task, articulatory suppression disproportionately affected the interference trials (i.e., related-no trials), which were the trials that contained semantic interference, and did not generally worsen performance on all trial types.

Taken together, findings from Experiment 1 show that articulatory suppression increases both PI and SI effects. One possibility is that articulatory suppression may reduce the availability of implicitly generated contextual tags that could be used to discern whether or not a probe's familiarity is due to its membership in the current memory set (Nairne, 2002). Such contextual tags need only be consulted when the item-specific and familiarity-based memory processes conflict, as they do on recent-no and related-no trials. Another possibility is that articulatory suppression may reduce the distinctiveness of current memoranda, lowering the signal-to-noise ratio between these items and those active due to prior study or spreading semantic activation. This reduction in distinctiveness may lead to increased interference from recent-no and lure probes, whereas "yes" probes may maintain sufficient activation to support timely recognition.

Our findings could have turned out much differently, however. For example, articulatory suppression could have also weakened representations of interfering items (from past trials, in the case of the recent-probes task, or semantically related associates in the short-term DRM task), which might have mitigated both interference effects. Instead, our findings show that the influence of articulatory suppression was disproportionally greater for interference trials on both tasks, suggesting some selectivity of the effects of articulatory suppression. These results thus offer support to the notion that articulatory suppression weakens the strength of current trial representations relative to interfering items, and not vice versa.

The parallel influence of articulatory suppression on PI and SI suggests that this manipulation similarly influences the quality of memory representations that must be consulted in order to overcome both forms of interference. Furthermore, the similarly disproportionate effects of articulatory suppression on the magnitude of interference originating from both temporal/episodic familiarity (recentprobes task) and semantic similarity (short-term DRM task) suggests that the resolution of PI and SI may recruit similar or shared cognitive control processes. In order to further explore this possibility, Experiment 2 examined the interaction of PI and SI more directly by having participants perform a task that contained both semantic and episodic interference.

\section{Experiment 2}

The presence of SI in short-term memory suggests that the semantic context of probe items is used in the recognition decision process. Related-no probes take longer to reject and are more likely to be falsely recognized because they are related in meaning to the items currently in short-term memory. If the semantic relationship between the memory set and the probe can induce interference, perhaps semantic contextual tags can be leveraged to reduce PI in cases where the semantic context of a recent-no probe is incongruent with that of the current memory set. In other words, items coming from a different semantic context than the current memoranda will have more distinct contextual retrieval cues, which should mitigate their interfering potential. By the same token, if the semantic contexts of the current memory set and a recent-no probe overlap, interference should increase because of the need to adjudicate both the semantic and episodic familiarity of this item. In this case, the contextual cues associated with current memoranda and past items will be more similar, and therefore retrieval will be more difficult. This logic is consistent with both contextual-cuing models (Nairne, 2002) and feature-based models (Nairne, 1990) of memory.

Experiment 2 was designed to examine this possibility using a paradigm that incorporated semantic categories into a standard recent-probes task. By incorporating factors that 
induce PI (episodic familiarity) and SI (semantic familiarity) into the same paradigm, this design allowed us to examine the interaction between these variables. Participants completed two versions of the recent-probes task, one that utilized memory sets in which all memoranda were exemplars of a single semantic category (e.g., all fruit words; the categorized version of the task), and one in which the memoranda were not (i.e., the standard recentprobes task). The categorized version included two forms of recent-no trials: recent-no_match trials, in which the recentno probe matched the semantic category of the current memory set, and recent-no_mismatch trials, in which the recent-no probes were from a different semantic category than the current memory set. We predicted that if semantic tags are utilized in the assessment of recent-no probes, we should find a Recency x Semantic Context interaction, such that interference would be increased for recent-no_match probes and reduced for recent-no_mismatch probes.

An additional advantage of this design is that it allowed us to independently assess SI effects by comparing trials in which nonrecent-no probes were members of the same semantic category as the current memoranda (nonrecentno_match trials) with trials in which they were not (nonrecent-no_mismatch trials). Note that the comparison of these trial types allows for the assessment of SI independent of PI, since neither contained words from the previous memory set. Previous work on the associative nature of semantic memory has demonstrated semantic priming using category exemplars (Collins \& Quillian, 1969; Huttenlocher \& Kubicek, 1983; Neely, 1976). We therefore predicted that the categorical relationship between memory items would be sufficient to induce spreading activation to unstudied category exemplars, thereby increasing their familiarity.

\section{Method}

\section{Participants}

Twenty-four participants (10 males; mean age 22.5 years) were recruited from the University of Michigan to participate in the study. All participants gave informed consent, as reviewed by the University of Michigan's Institutional Review Board. Participants were paid \$15 per hour for their participation, plus bonuses for fast and accurate responding throughout the experiment. Bonus scores were calculated as in Experiment 1.

\section{Design/procedure}

We used a modified version of the recent-probes task (Exp. 1) to jointly assess the effects of PI and SI within the same paradigm. To incorporate semantic familiarity into the recent-probes task, memory sets were drawn from two semantic categories, fruits and countries. There were 50 words used as memoranda: 25 fruit words and 25 country words. In the categorized version of the task (see Fig. 3), memory sets consisted of either all fruit words or all country words, with the category alternating in a random fashion. This procedure allowed us to examine (1) instances in which the semantic category remained the same over two consecutive trials, and (2) instances in which the semantic category changed from trial to trial. In the mixed version of the task, we used the same words, but memory sets contained a mixture of fruit and country words, making categorization of any single memory set impossible. In this task, probes were drawn equally from both semantic categories.

The total number of trials was 256 for both the mixed (MIX) and categorized (CAT) versions of the task. In the MIX task, there were 128 yes_mixed trials, 64 recentno_mixed trials, and 64 nonrecent-no_mixed trials. In the CAT task, there were 128 yes_match trials, 32 nonrecentno_match trials, 32 nonrecent-no_mismatch trials, 32 recent-no_match trials, and 32 recent-no_mismatch trials. Across all trials in the CAT version, half of all memory set items and half of all probe items were drawn from each semantic category. Experiment 2 utilized a within-subjects design, with all participants completing both versions of the task. Task order was counterbalanced across participants.

\section{Results and discussion}

Experiment 2 demonstrated interactive effects between the variables that induced PI and SI. When recent-no probes were taken from the same semantic category as the current memory set, we found that PI and SI interacted reliably. In addition, we found that semantic context could reliably mitigate PI if recent-no probes were drawn from a different semantic category than the current memory set.

Mean accuracy (displayed as hits and false alarms) and median RTs for correct responses to each probe type in each task are presented in Fig. 4. Repeated measures ANOVAs were conducted to assess the effects of recency (recent vs. nonrecent), semantic context (mixed, mismatch, or match), and task order (MIX first vs. CAT first) on probe accuracy and RT. With respect to "no" probes, we found main effects of both recency and context on false alarm rates $[F(1,22)=$ $32.94, \eta^{2}=.60$, and $F(2,21)=51.03, \eta^{2}=.43$, respectively; $p<.001$ for both] and on RTs for correct rejections $\left[F(1,22)=51.03, \eta^{2}=.70\right.$, and $F(2,21)=10.39$, $\eta^{2}=.32$, respectively; $p<.001$ for both]. There was no main effect of semantic context on hits or RTs for correct recognitions, indicating that participants were equally fast and accurate at recognizing memoranda from both categorized and mixed stimulus sets.

Central to our hypothesis, we found Context $\mathrm{x}$ Recency interactions with respect to both false alarm rates, $F(5,18)=$ 
Fig. 3 The categorized recentprobes (RP) task utilized in Experiment 2. Memoranda on each trial consisted of either four fruit words or four country words. The category membership of the memory sets was unpredictable, such that adjacent trials could be drawn from either the same category (e.g., fruitfruit) or different categories (e. g., fruit-country). This procedure allowed us to examine two forms of recent-no probes, including recent-no_match probes, which were recently studied items from the same category presented in the current memory set, and recent-no mismatch probes, which were recently studied items from a category different than the one presented in the current memory set. Example trials are shown above. In the mixed version of the task, memory sets contained a random mixture of words from both categories. The first row represents Trial 1, the second row Trial 2, and so forth

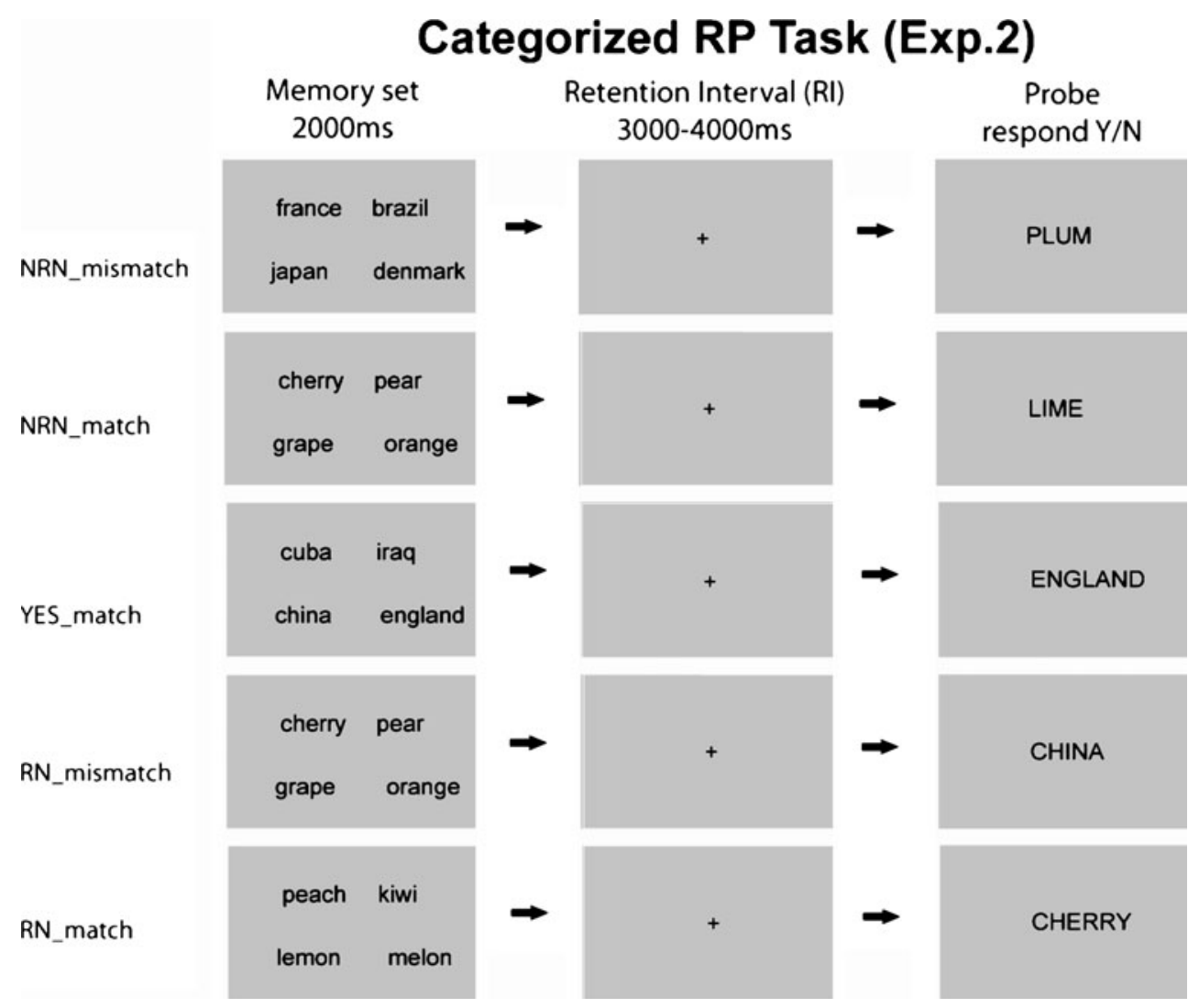

9.92, $p<.001, \eta^{2}=.31$, and correct-rejection RTs, $F(5,18)=$ $7.86, p<.001, \eta^{2}=.26$, indicating that PI reliably varied between mixed, matched, and mismatched semantic contexts. In order to test our prediction that interference would increase when recent-no probes were drawn from the same semantic category as the current memory set, we conducted planned comparisons of RTs and false alarm rates for recent-no_match versus recent-no_mixed trials. Findings showed a significant, $5 \%$ increase in false alarms, $t(23)=$ $-3.96, p<.001, d=0.74$, and a 28 -ms increase in RTs, $t(23)=2.23, p<.05, d=1.29$, associated with recentno_match versus recent-no_mixed probes. We also found decreased interference when recent-no probes were drawn from a different semantic category than the memoranda. Participants made 3\% fewer false alarms, $t(23)=2.63, p<$ $.05, d=0.63$, and were $31 \mathrm{~ms}$ faster in correct rejections, $t(23)=2.90, p<.05, d=0.31$, for recent-no_mismatch versus recent-no_mixed probes.

We assessed SI independently of PI by comparing RTs and false alarm rates for nonrecent-no_match and nonrecent-no_mismatch trials using paired $t$ tests. The results showed a $2 \%$ increase in false alarms to nonrecentno probes from the same semantic category, $t(23)=-2.28$, $p<.05, d=0.53$, as well as an $18-\mathrm{ms}$ increase in correctrejection RTs for these items, $t(23)=2.27, p<.05, d=$ 0.18 , relative to nonrecent-no items from a different semantic category. These findings indicate that our task was successful in producing SI effects that were independent of the effects of PI. ${ }^{2}$

Taken together, the results from Experiment 2 suggest that the semantic relationship between recent-no probes and memory set items changed the extent to which these items induced PI. Consistent with our predictions, we observed an interaction between probe recency and semantic context, such that interference increased when recent-no probes shared a semantic context with memoranda and decreased when the semantic contexts were distinct. These findings suggest that the resolution of both semantic and episodic forms of interference may rely on similar processes and resources. Recent-no_match probes, which are both episodically and semantically familiar, induce more interference than recent-no_mixed probes, which have episodic familiarity but share no informative semantic relationship with the memory set. Recentno_mismatch probes, which are episodically familiar but semantically distinct from the memoranda, induce the least amount of PI.

\footnotetext{
$\overline{2}$ There was also a trend in which nonrecent-no match trials had greater RTs (by $15 \mathrm{~ms}$ ) than nonrecent-no_mixed trials, $t(23)=1.7, p=$ .103 .
} 

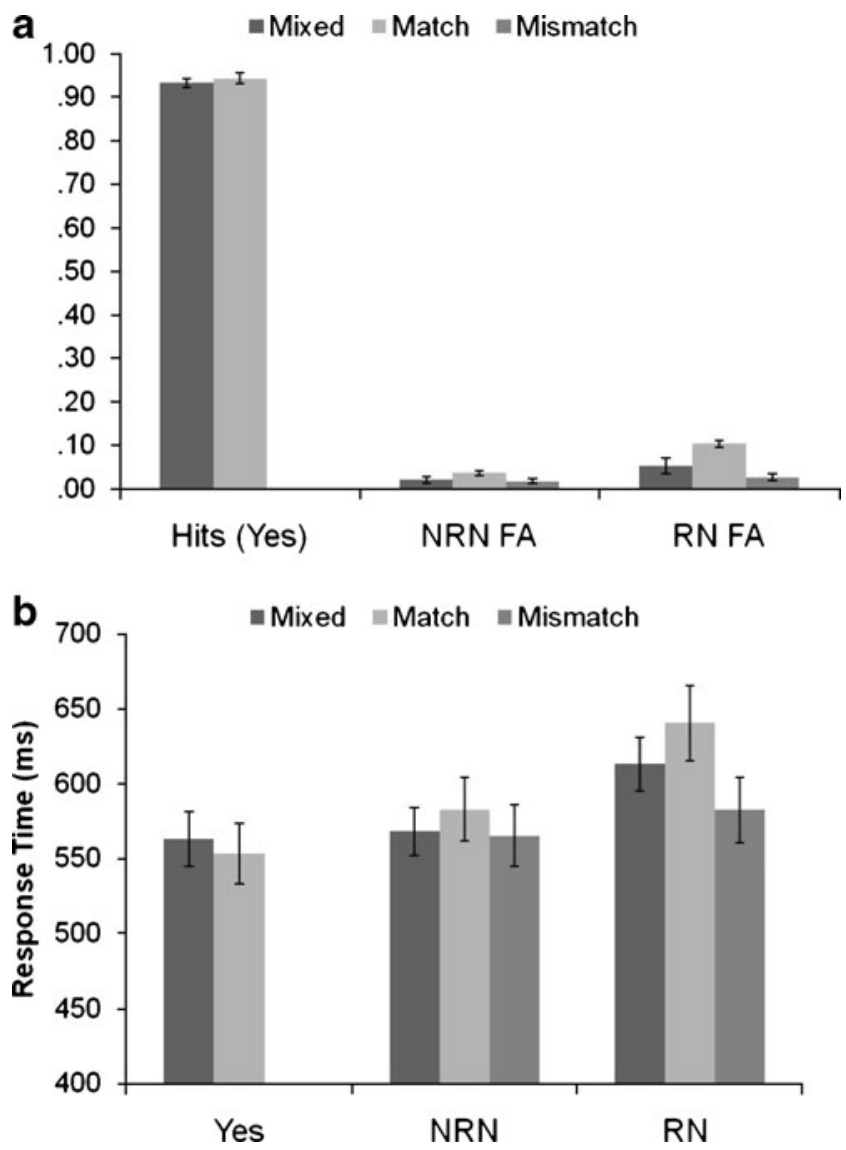

Fig. 4 Mean accuracy (hits and false alarms) and median RTs plotted by the context in which probes appeared. On mixed trials, which were blocked, memory sets included a mixture of words from two semantic categories (fruits and countries), and recent-no (RN) probes were drawn from each category with equal probability. On both mismatch and match trials (which appeared in the categorized block), memory sets contained items from only one semantic category, which alternated randomly from trial to trial. Both $\mathrm{RN}$ and nonrecent-no $(\mathrm{NRN})$ probes were drawn with equal probability from either the same category as the current memoranda (match trials) or from the other category (mismatch trials)

\section{General discussion}

To date, most studies have investigated the effects of proactive and semantic interference in short-term memory independently of each other. The purpose of the present work was to examine these effects jointly and to assess whether common or shared cognitive processes may be recruited to resolve the interference induced by both episodic and semantic familiarity. Experiment 1 demonstrated that the requirement to engage in articulatory suppression during the retention interval of an item recognition task (i.e., the recent-probes task or the shortterm DRM task) increased interference induced by the need to reject both recently studied items (PI) and items that had never been studied but that were related in meaning to current memoranda (SI). That articulatory suppression increased both PI and SI in similar ways (i.e., disproportionately affecting trials that had either proactive or semantic interference) suggests that this manipulation interferes with the processing required for the resolution of both forms of interference. Furthermore, this work replicates our previous findings (Atkins \& Reuter-Lorenz, 2008) by showing that SI can be induced using a short-term memory paradigm in which lure (related-no) probes were never studied and in which the semantic theme of each memory set was unique.

Experiment 2 specifically investigated the interactions between PI and SI by incorporating both episodic and semantic familiarity into a single paradigm. Consistent with our predictions, we found an interaction between the semantic context of a recent-no probe and the amount of interference produced. This suggests that vulnerability to PI changes depending on the meaningful relationship between previously studied information and the items currently in memory. Interestingly, PI was reliably reduced when recent-no probes were drawn from a semantic context distinct from current memoranda, suggesting that semantic cues can be leveraged to reduce interference from episodic familiarity. Despite this reduction, however, significant residual PI remained, suggesting that even when recent-no probes could feasibly be rejected on the basis of semantic information alone, this was not sufficient to eradicate the pervasive effects of episodic familiarity.

Engagement in both the recent-probes task and the shortterm DRM task requires participants to negotiate the familiarity of critical probe items (recent-no or related-no probes) in the service of accurate task performance. In the recent-probes task, recent-no probes are activated due to their recent status as memoranda, while in the short-term DRM task, related-no probes presumably become active at encoding via spreading activation through semantic networks (Collins \& Loftus, 1975; Roediger, McDermott, \& Robinson, 1998; Underwood, 1965). One possibility is that both the episodic and semantic contexts of critical probes may promote source memory confusion, increasing the likelihood that such probes will be misattributed to the current memory set (Nelson, Reuter-Lorenz, Sylvester, Jonides, \& Smith, 2003).

This account is consistent with feature-based models (Nairne, 1990) and with contextual-cuing models (Nairne, 2002) of short-term remembering. According to these models, successful memory retrieval relies on accurate recombination of activated cues associated with various features of the memoranda, such as episodic, semantic, lexical, and phonological features. A reduction in the distinctiveness of episodic or semantic features, or contextual cues that distinguish probes from the memoranda, will induce interference. These models could account for the 
pattern of interference effects we observed by predicting an increase in interference as retrieval cues become less distinct. For probes related in meaning to memoranda, there is overlap between the semantic cues associated with each; for recent-no probes, there is overlap between episodic cues. When a recent-no probe shares a semantic context with memoranda, both semantic and episodic cues overlap, leading to increased interference. By the same token, when contextual cues become more distinct, interference is mitigated.

While feature-based and contextual-cuing models (Nairne, 1990, 2002) can, in principle, account for the presence of an interaction of PI and SI, they remain silent about the necessity of a specific cognitive mechanism dedicated to interference resolution. The assumption of such a mechanism is common in the neuroimaging literature, in which many researchers (including ourselves) investigating interference effects in short-term retrieval have proposed a distinct interference resolution mechanism that is engaged when one is faced with episodic or semantic interference (e.g., Badre \& Wagner, 2005). This common assumption is grounded in a kind of subtraction logic: Additional cognitive mechanisms are posited to account for increased neural activation for one condition versus another.

However, the existence of a distinct interference resolution mechanism is not a conclusion forced by the data. In fact, more recent research has conceptualized interference resolution as the requirement to engage in greater memory selection/search processes (Nee \& Jonides, 2009), which are lengthened when stimuli impose episodic or semantic interference. One promising framework that does not require a distinct resolution mechanism is signal detection theory (SDT; Green \& Swets, 1966; Macmillan \& Creelman, 2005). Applying SDT to the current tasks, target probe items might be represented as noisy codes in a two-dimensional space in which one dimension represents the match to the current episodic context, and another dimension represents the match to the current semantic context. "Yes" trials would be high on both semantic and episodic similarity, but so would (to a lesser extent) recent-no_match trials. The decision process is thus construed as a problem of discrimination under conditions of noise, and interference is simply a descriptive label given to conditions in which the discrimination is more difficult. Such an account could be similar to a biasedcompetition account for overcoming interference that was proposed by Jonides and Nee (2006), but framing the model in SDT terms provides a more principled theoretical grounding. ${ }^{3}$ Although we presently favor a SDT framework

\footnotetext{
${ }^{3}$ SDT models and biased-competition models may differ in how they assume data to be distributed. SDT models assume normal distributions, while many biased-competition models may yield more skewed distributions of the data.
}

over theories that assume an additional and separate inference resolution process, we leave development and testing of such a framework for future work.

Regarding the nature of SI and PI, some additional comments are warranted. Although we are claiming that PI and SI are distinct forms of interference, there may be some concern that the two are indistinguishable. For example, in the short-term DRM paradigm of Experiment 1, participants studied a list of words that were all related to a common theme word. By one popular account (see Gallo, 2006, for a review), participants automatically generate this theme word, which produces false memories, and presumably SI. Stated another way, participants may generate this theme word close in time to the study of the current memoranda. Given the potential temporal closeness of study and the theme word generation, one might argue that interference that is later produced by the need to reject this theme word could actually be described as a self-generated PI effect. We have three comments on this point. First, the temporal-episodic origins of PI and SI differ. In the recentprobes task, PI is induced by words encoded from the prior trial. In contrast, the SI effect is induced by associations with memoranda on the current trial, and therefore is not "proactive." Similarly, unlike PI, which builds up over trials of semantically related items in the classic release-from-PI effect (Wickens, 1970), the lists used in Experiment 1 were unique on each trial, in that semantic themes never repeated. Because the trials were semantically distinct, interference from one trial to the next was less likely. Second, the theme word likely interfered not because it was generated close in time to the memoranda, but because it was semantically related to the memoranda and thus confusable. Indeed, pilot data from our lab (Sodhi, Atkins, \& Reuter-Lorenz, 2010) indicate that the magnitude of SI depends on the associative strength between the memoranda and the theme word. Again, this differs from the recent-probes task, in which items interfere because they were encoded close in time, regardless of their semantic relationship to the current trial's stimuli; our results from Experiment 2, however, do suggest that interference can be accentuated if there is a semantic relationship between previous items and the current trial. Third, when we explored the time course of PI and SI effects over the duration of Experiment 1, we found that the SI effects increased over blocks, but the PI effects remained constant. The fact that there were reliable differences in the time courses of these effects suggests that they were not identical. While PI and SI may not be mutually exclusive constructs, the aforementioned ideas and data suggest that there are distinguishing characteristics between these two forms of interference.

This work builds on the work of Oztekin, Curtis, and McElree (2008), who also studied the relationship between PI and SI. Moreover, the present study extends those results 
by independently assessing both forms of interference and by examining SI produced within individual trials, as opposed to that which builds up across multiple trials. The distinction between within-trial and across-trial interference may explain why those authors failed to find interactive effects of PI and SI such as those reported here.

The present research also builds on earlier investigations by Wickens, Moody, and Dow (1981), who demonstrated increased short-term memory search time associated with the buildup of PI induced by repeated exposure to memory lists from the same semantic category. This study, however, did not incorporate episodic recency, and therefore could not test the interaction between interference induced by episodic and semantic familiarity.

The present work offers a unique contribution to the literature by showing that semantic context can change the degree to which we are vulnerable to PI, increasing this vulnerability when semantic contexts overlap and decreasing (though not eliminating) it when semantic contexts are distinct. Taken together, our findings are consistent with the notion that shared processes are recruited in the service of the resolution of multiple forms of interference (Nelson, Reuter-Lorenz, Persson, Sylvester, \& Jonides, 2009; Oztekin et al., 2008), and they suggest these shared processes may be best characterized in terms of signal detection operations that mediate mnemonic decisions at retrieval. In addition, this work may contribute to the burgeoning field of research focused on the development of cognitive training regimens that may improve memory performance (Berman, Jonides, \& Kaplan, 2008; Jaeggi, Buschkuehl, Jonides, \& Perrig, 2008). Uncovering the circumstances in which interference is mitigated and enhanced and identifying shared cognitive processing across tasks may be crucial to the development of improved interventions, as well as to our increased understanding of the cognitive control of memory.

Acknowledgments This work was supported in part by an NSF grant to J.J., an NIH grant to J.J., an NSF Graduate Fellowship to M.G.B., and an NRSA fellowship to A.S.A. We thank Laura Hieber, Courtney Behnke, and Halle Zucker for help with data collection. The first two authors contributed equally to this work.

\section{References}

Anderson, J. R., Bothell, D., Byrne, M. D., Douglass, S., Lebiere, C., \& Qin, Y. (2004). An integrated theory of mind. Psychological Review, 111, 1036-1060.

Atkins, A. S., \& Reuter-Lorenz, P. A. (2008). False working memories? Semantic distortion in a mere 4 seconds. Memory \& Cognition, 36, 74-81.

Atkinson, R. C., \& Shiffrin, R. M. (1968). Human memory: A proposed system and its control processes. In K. W. Spence \& J. T. Spence (Eds.), The psychology of learning and motivation (Vol. 2, pp. 89-195). New York: Academic Press.

Baddeley, A. D., \& Hitch, G. J. (1974). Working memory. In G. H. Bower (Ed.), The psychology of learning and motivation:
Advances in research and theory (Vol. 8, pp. 47-89). New York: Academic Press.

Baddeley, A. D., Thomson, N., \& Buchanan, M. (1975). Word length and structure of short-term memory. Journal of Verbal Learning and Verbal Behavior, 14, 575-589.

Badre, D., \& Wagner, A. D. (2005). Frontal lobe mechanisms that resolve proactive interference. Cerebral Cortex, 15, 2003-2012.

Berman, M. G., Jonides, J., \& Kaplan, S. (2008). The cognitive benefits of interacting with nature. Psychological Science, 19, 1207-1212.

Berman, M. G., Jonides, J., \& Lewis, R. L. (2009). In search of decay in verbal short-term memory. Journal of Experimental Psychology. Learning, Memory, and Cognition, 35, 317-333.

Collins, A. M., \& Loftus, E. F. (1975). A spreading-activation theory of semantic processing. Psychological Review, 82, 407-428.

Collins, A. M., \& Quillian, M. R. (1969). Retrieval time from semantic memory. Journal of Verbal Learning and Verbal Behavior, 8, 240-247.

Conrad, R. (1964). Acoustic confusions in immediate memory. British Journal of Psychology, 55, 75-84.

Cowan, N. (1988). Evolving conceptions of memory storage, selective attention, and their mutual constraints within the human information processing system. Psychological Bulletin, 104, 163-191.

Cowan, N. (1995). Attention and memory: An integrated framework. New York: Oxford University Press.

Cowan, N. (2000). The magical number 4 in short-term memory: A reconsideration of mental storage capacity. The Behavioral and Brain Sciences, 24, 87-185.

Gallo, D. A. (2006). Associative illusions of memory: False memory research in DRM and related tasks. New York: Psychology Press.

Green, D. M., \& Swets, J. A. (1966). Signal detection theory and psychophysics. New York: Wiley.

Hancock, T., \& Hicks, J. (2002). Backward associative strength determines source attributions given to false memories. Psychonomic Bulletin \& Review, 9, 807-815.

Huttenlocher, J., \& Kubicek, L. F. (1983). The source of relatedness effects on naming latency. Journal of Experimental Psychology. Learning, Memory, and Cognition, 9, 486-496.

Jaeggi, S. M., Buschkuehl, M., Jonides, J., \& Perrig, W. J. (2008). Improving fluid intelligence with training on working memory. Proceedings of the National Academy of Sciences, 105, 6829-6833.

Jonides, J., Lewis, R. L., Nee, D. E., Lustig, C. A., Berman, M. G., \& Moore, K. S. (2008). The mind and brain of short-term memory. Annual Review of Psychology, 59, 193-224.

Jonides, J., \& Nee, D. E. (2006). Brain mechanisms of proactive interference in working memory. Neuroscience, 139, 181-193.

Jonides, J., Smith, E. E., Marshuetz, C., Koeppe, R. A., \& ReuterLorenz, P. A. (1998). Inhibition in verbal working memory revealed by brain activation. Proceedings of the National Academy of Sciences, 95, 8410-8413.

Keppel, G. (1968). Retroactive and proactive inhibition. In T. R. Dixon \& D. L. Horton (Eds.), Verbal behavior and general behavior theory (pp. 172-213). Englewood Cliffs, NJ: Prentice Hall.

Laughery, K. R., \& Harris, G. J. (1970). Visual and auditory intrusion errors in short-term memory. Journal of Experimental Psychology, 83, 101-106.

Levy, B. A. (1971). Role of articulation in auditory and visual shortterm memory. Journal of Verbal Learning and Verbal Behavior, $10,123-132$.

Lewandowsky, S., Geiger, S. M., \& Oberauer, K. (2008). Interferencebased forgetting in verbal short-term memory. Journal of Memory and Language, 59, 200-222.

Logie, R. H., Della Sala, S., Wynn, V., \& Baddeley, A. D. (2000). Visual similarity effects in immediate verbal serial recall. The Quarterly Journal of Experimental Psychology, 53A, 626-646. 
Macmillan, N. A., \& Creelman, C. D. (2005). Detection theory: A user's guide. Mahwah, $\mathrm{NJ}$ : Erlbaum.

McElree, B. (2001). Working memory and focal attention. Journal of Experimental Psychology. Learning, Memory, and Cognition, 27, $817-835$.

Monsell, S. (1978). Recency, immediate recognition memory, and reaction-time. Cognitive Psychology, 10, 465-501.

Nairne, J. S. (1990). A feature model of immediate memory. Memory \& Cognition, 18, 251-269.

Nairne, J. S. (2002). Remembering over the short-term: The case against the standard model. Annual Review of Psychology, 53, 53-81.

Nee, D. E., \& Jonides, J. (2009). Common and distinct neural correlates of perceptual and memorial selection. Neuroimage, 45, 963-975.

Neely, J. H. (1976). Semantic priming and retrieval from lexical memory: Evidence for facilitatory and inhibitory processes. Memory \& Cognition, 4, 648-654.

Nelson, J. K., Reuter-Lorenz, P. A., Persson, J., Sylvester, C. Y., \& Jonides, J. (2009). Mapping interference resolution across task domains: A shared control process in left inferior frontal gyrus. Brain Research, 1256, 92-100.

Nelson, J. K., Reuter-Lorenz, P. A., Sylvester, C. Y., Jonides, J., \& Smith, E. E. (2003). Dissociable neural mechanisms underlying response-based and familiarity-based conflict in working memory. Proceedings of the National Academy of Sciences, 100, 1117111175.

Nichols, E. A., Kao, Y.-C., Verfaellie, M., \& Gabrieli, J. D. E. (2006). Working memory and long-term memory for faces: Evidence from fMRI and global amnesia for involvement of the medial temporal lobes. Hippocampus, 16, 604-616.

Oberauer, K. (2002). Access to information in working memory: Exploring the focus of attention. Journal of Experimental Psychology. Learning, Memory, and Cognition, 28, 411-421.

Oztekin, I., Curtis, C. E., \& McElree, B. (2008). The medial temporal lobe and the left inferior prefrontal cortex jointly support interference resolution in verbal working memory. Journal of Cognitive Neuroscience, 21, 581-593.

Postman, L. (1961). Extra-experimental interference and retention of words. Journal of Experimental Psychology, 61, 97-110.

Postman, L., \& Underwood, B. J. (1973). Critical issues in interference theory. Memory \& Cognition, 1, 19-40.
Ranganath, C., \& Blumenfeld, R. S. (2005). Doubts about double dissociations between short- and long-term memory. Trends in Cognitive Sciences, 9, 374-380.

Ranganath, C., \& D'Esposito, M. (2005). Directing the mind's eye: Prefrontal, inferior and medial temporal mechanisms for visual working memory. Current Opinion in Neurobiology, 15, 175182.

Roediger, H. L., III, \& McDermott, K. B. (1995). Creating false memories: Remembering words not presented in lists. Journal of Experimental Psychology. Learning, Memory, and Cognition, 21, 803-814.

Roediger, H. L., III, McDermott, K. B., \& Robinson, K. J. (1998). The role of associative processes in producing false memories. In M. Conway, S. Gathercole, \& C. Cornoldi (Eds.), Theories of memory II (pp. 187-245). Hove, U.K.: Psychological Press.

Roediger, H. L., III, Watson, J. M., McDermott, K. B., \& Gallo, D. A. (2001). Factors that determine false recall: A multiple regression analysis. Psychonomic Bulletin \& Review, 8, 385-407.

Sodhi, P., Atkins, A. S., \& Reuter-Lorenz, P. A. (2010). The effect of backward associative strength on false working memories and the semantic interference effect. Unpublished manuscript.

Underwood, B. J. (1965). False recognition produced by implicit verbal responses. Journal of Experimental Psychology, 70, 122129.

Underwood, B. J., \& Schultz, R. W. (1960). Meaningfulness and verbal learning. Philadelphia: Lippincott.

Verhaeghen, P., Cerella, J., \& Basak, C. (2004). A working memory workout: How to expand the focus of serial attention from one to four items in 10 hours or less. Journal of Experimental Psychology. Learning, Memory, and Cognition, 30, 1322-1337.

Wickens, D. D. (1970). Encoding categories of words: Empirical approach to meaning. Psychological Review, 77, 1-15.

Wickens, D. D., Dalezman, R. E., \& Eggemeier, F. T. (1976). Multiple encoding of word attributes in memory. Memory \& Cognition, 4, 307-310.

Wickens, D. D., Moody, M. J., \& Dow, R. (1981). The nature and timing of the retrieval process and of interference effects. Journal of Experimental Psychology: General, 110, 1-20.

Wixted, J. T. (2005). A theory about why we forget what we once knew. Current Directions in Psychological Science, 14, 6-9. 